\section{Original Article}

\section{Corresponding Author}

Kota Watanabe

(iD https://orcid.org/0000-0002-4830-4690

Department of Orthopaedic Surgery, Keio

University School of Medicine,

Shinanomachi 35, Shinjuku-ku, Tokyo

160-8582, Japan

Email: kw197251@keio.jp

Received: June 17, 2020

Revised: August 22, 2020

Accepted: September 20, 2020

\section{(c) (i) (8)}

This is an Open Access article distributed under the terms of the Creative Commons Attribution Non-Commercial License (https://creativecommons.org/licenses/by-nc/4.0/) which permits unrestricted non-commercial use, distribution, and reproduction in any medium, provided the original work is properly cited.

Copyright (c) 2021 by the Korean Spinal Neurosurgery Society

\title{
Metabolic Syndrome is a Predisposing Factor for Diffuse Idiopathic Skeletal Hyperostosis
}

\author{
Eijiro Okada ${ }^{1,2}$, Shinichi Ishihara ${ }^{2,3}$, Koichiro Azuma ${ }^{4}$, Takehiro Michikawa, \\ Satoshi Suzuki ${ }^{1,2}$, Osahiko Tsuji ${ }^{1,2}$, Satoshi Nori ${ }^{1,2}$, Narihito Nagoshi ${ }^{1,2}$, Mitsuru Yagi ${ }^{1,2}$, \\ Michiyo Takayama $^{6}$, Takashi Tsuji ${ }^{2,7}$, Nobuyuki Fujita ${ }^{2,8}$, Masaya Nakamura ${ }^{1,2}$, \\ Morio Matsumoto ${ }^{1,2}$, Kota Watanabe ${ }^{1,2}$ \\ ${ }^{1}$ Department of Orthopaedic Surgery, Keio University School of Medicine, Tokyo, Japan \\ ${ }^{2}$ Keio Spine Research Group, Tokyo, Japan \\ ${ }^{3}$ Department of Orthopaedic Surgery, Ota Memorial Hospital, Oshimasho, Ota City, Japan \\ ${ }^{4}$ Institute for Integrated Sports Medicine, Keio University School of Medicine, Tokyo, Japan \\ ${ }^{5}$ Department of Environmental and Occupational Health, Toho University, Tokyo, Japan \\ ${ }^{6}$ Center for Preventive Medicine, Keio University Hospital, Tokyo, Japan \\ ${ }^{7}$ Department of Orthopaedic Surgery, National Hospital Organization Tokyo Medical Center, Tokyo, Japan \\ ${ }^{8}$ Department of Orthopaedic Surgery, Fujita Health University, Toyoake-shi, Japan
}

Objective: Diffuse idiopathic skeletal hyperostosis (DISH) causes spinal ankylosis, which can result in patients suffering specific spinal fractures that lead to a reduction in the activities of daily life in older patients. Currently, DISH is associated with diabetes mellitus and cardiovascular disease; however, the association between DISH and metabolic syndrome has not been established. The purpose of this study was to investigate a potential association between DISH and metabolic syndrome.

Methods: We retrospectively reviewed clinical data from consecutive subjects undergoing the musculoskeletal health medical checkups, and enrolled 327 subjects (174 men and 153 women; mean, 63.4 \pm 13.7 -years). Subjects who had spinal ankylosis at least 4 contiguous vertebral bodies were classified as the DISH group $(n=39)$ while the others were part of the non-DISH group $(\mathrm{n}=288)$. The definition of the metabolic syndrome comes from diagnostic criteria used by the Japanese Society for Internal Medicine. Age, sex, body max index (BMI), hematological evaluation, blood pressure, presence of metabolic syndrome, the visceral fat area on abdominal computed tomography, and spinal epidural lipomatosis (SEL) on magnetic resonance imaging were evaluated.

Results: Compared to the non-DISH group, in the DISH group, mean age (DISH group, 74.3 years; non-DISH group, 1.9 years; $\mathrm{p}<0.001$ ), male prevalence were higher (DISH group, $82.1 \%$; non-DISH group, $49.3 \%$; $\mathrm{p}<0.001$ ), and BMI was greater (DISH group, 24.8; non-DISH group, $23.0 ; \mathrm{p}=0.006)$. the metabolic syndrome was more frequently observed in DISH group $(28.9 \%)$ than in the non-DISH group $(16.0 \%)(p=0.045)$. The visceral fat area was significantly larger in the DISH group than in the non-DISH group (DISH group, $130.7 \pm 58.2 \mathrm{~cm}^{2}$; Non-DISH group, $89.0 \pm 48.1 \mathrm{~cm}^{2}$; $\left.\mathrm{p}<0.001\right)$. The prevalence of SEL was similar between the 2 groups $(10.3 \%$ in the DISH group vs. $8.7 \%$ in the nonDISH group; $\mathrm{p}=0.464$ ). Poisson regression analysis revealed that the metabolic syndrome was significantly associated with DISH with odds ratio of 2.0 (95\% confidence interval, 1.0 $3.7 ; \mathrm{p}=0.004)$.

Conclusion: Metabolic syndrome was significantly associated with DISH. Our data showed metabolic syndrome is potentially related to DISH.

Keywords: Diffuse idiopathic skeletal hyperostosis, Metabolic syndrome, Spinal epidural lipomatosis, Body max index, Blood pressure, Spinal ankylosis 


\section{INTRODUCTION}

The concept of diffuse idiopathic skeletal hyperostosis (DISH) was first suggested by Resnick et al. ${ }^{1}$ in 1975 . DISH is a noninfuriately condition with systemic skeletal ankylosis that mainly affects the T7-11 region of the thoracic spine at onset but tends to extend in the cephalad and caudal direction with age. ${ }^{2} \mathrm{DISH}$ can negatively affect activities of daily life in older patients because it can cause clinical problems such as low-back pain, limitation in spinal movement, ${ }^{3}$ dysphasia, ${ }^{4}$ spinal fracture after minor trauma, ${ }^{5-7}$ and poor outcomes after surgery for lumbar degenerative disease. ${ }^{8-10}$ To date, previous reports have identified certain factors associated with DISH, such as male sex, ${ }^{11,12}$ old age ( $>50$ years), ${ }^{11-13}$ synthetic retinoid use, ${ }^{14}$ ethnic factors (less common in the black, Native-American, and Asian), ${ }^{13,15}$ and genetic factors (COL6A1). ${ }^{16}$

Furthermore, metabolic abnormalities, ${ }^{17}$ diabetes mellitus $(\mathrm{DM}){ }^{18,19}$ and cardiovascular diseases ${ }^{20}$ have also been previously reported to have a potential association with DISH. In 1996, Vezyroglou et al. ${ }^{19}$ compared 100 patients with DISH with 100 patients without DISH who were matched for age, sex, and body mass index and found that metabolic disturbances such as DM, dyslipidemia (DL), and hyperuricemia were confined to the DISH group.

Metabolic syndrome is characterized by metabolic abnormalities, including insulin resistance and the associated abdominal obesity, hyperglycemia, hyperinsulinemia, DL, and hypertension. ${ }^{21,22}$ Previous studies indicated that metabolic syndrome increased the risk of type $2 \mathrm{DM}$, cardiovascular events, and death. The concept of metabolic syndrome was first reported in the United States in 2001 and the pathophysiology of metabolic syndrome has been revised after additional research. According to the National Health and Nutrition Examination Survey ${ }^{23}$ the prevalence of metabolic syndrome in the United States over 20 years is approximately $31.9 \%$ (30.6\% in men and $33.2 \%$ in women). Recently, the incidence of metabolic syndrome is increasing, even in developing countries, due to Western diet patterns.

Hence, metabolic disturbances seem to have an impact on the development of DISH. However, as DISH remains insufficiently investigated and understood, its definitive etiology is still unclear. Therefore, the purpose of this study was to elucidate the association between DISH and the metabolic syndrome.

\section{MATERIALS AND METHODS}

We obtained approval from the Institutional Review Board
(IRB) of Keio University School of Medicine (IRB number: 20160199) before initiating this retrospective cross-sectional study, and the study was a part of the musculoskeletal health checkup studies. ${ }^{24,25}$ Study subjects were notified of this study's opt-out option. We retrospectively identified 333 consecutive participants who underwent a musculoskeletal checkup in addition to a regular medical checkup from 2012 to 2016. The musculoskeletal checkup was voluntary and was not organized by the government or by a company. The cost of the musculoskeletal checkup was charged to the individual. Public medical insurance was not applied to this musculoskeletal medical checkup. Inclusion criteria for the study were participants: (1) who had the musculoskeletal checkup, (2) who are at least 20 years old, and (3) who have a complete dataset. The exclusion criteria for this study were participants: (1) who had a history of ankylosing spondylitis and (2) who did not consent to be in the study. Among these, 5 were excluded because of missing data, and we excluded one more because of a history of ankylosing spondylitis. Thus, 327 subjects were included in the study that comprised 174 men and 153 women with a mean age of $63.4 \pm 13.7$ years (range, 24-89 years) at the time of investigation. The presence of DISH was evaluated based on findings seen in the lateral and the anteroposterior aspects of spine radiographs. Subjects were diagnosed with DISH and classified into the DISH group $(n=39)$ if they had spinal ankylosis with flowing ossification along the anterolateral aspect of at least 4 contiguous vertebral bodies ${ }^{1}$; otherwise, they were categorized non-DISH group $(n=288)$. Variables such as waist circumference, systolic blood pressure (DBP), and diastolic blood pressure (DBP) were measured, and blood and urine assessments and serial radiographs of the thoracic spine, lumbar spine, abdominal computed tomography (CT), and lumbar spine magnetic resonance imaging (MRI) were performed.

In the abdominal CT, the visceral fat area (VFA) was measured at the level of the navel (Aquilion CXL, Toshiba Medical Systems Corp., Tochigi, Japan). Digital Imaging and Communication in Medicine data were analyzed using the sliceOmatic software (TomoVision, Magog, Quebec, Canada) ${ }^{25}$ Details of lumbar spine MRI acquisition have been described elsewhere by Ishihara et al. ${ }^{25}$ The anterior-posterior (AP) distance ratio of epidural fat was measured on T2-weighted axial images, and spinal epidural lipomatosis (SEL) was defined as $0.6 \mathrm{AP}$ distance ratio of epidural fat in at least one lumbar level. ${ }^{26}$ Additionally, we retrieved the results of blood and urine analyses, including high-density lipoprotein cholesterol (HDL-C), lowdensity lipoprotein cholesterol (LDL-C), triglycerides (TG), ad- 
iponectin, fasting blood glucose, glycated hemoglobin, insulin, homeostasis model assessment as an index of insulin resistance, urine microalbumin, high sensitivity C-reactive protein, and Btype natriuretic peptide. All blood samples were collected under overnight fasting conditions. The diagnostic criteria used for the metabolic syndrome were those defined by the Japanese Society for Internal Medicine ${ }^{27}$ and are as follows: VFA $\geq 100$ $\mathrm{cm}^{2}$ with any 2 of the following conditions: (1) TG $\geq 150 \mathrm{mg} /$
$\mathrm{dL}$ and/or HDL-C $<40 \mathrm{mg} / \mathrm{dL}$, (2) $\mathrm{SBP} \geq 130 \mathrm{mmHg}$ and/or DBP $\geq 85 \mathrm{mmHg}$, or (3) fasting plasma glucose $\geq 110 \mathrm{mg} / \mathrm{dL}$.

We examined the association between categorical variables and the presence of DISH using the chi-squared test. For the comparison of continuous variables between the DISH and nonDISH groups, we assumed no clear violation of the normality assumption and used Student t-test. We applied a Poisson regression model to examine a possible association between DISH

Table 1. Comparison between DISH (D) and non-DISH (N) groups

\begin{tabular}{|c|c|c|c|}
\hline Variable & $D$ group $(n=39)$ & $N$ group $(n=288)$ & p-value \\
\hline Age (yr) & $74.3 \pm 8.6$ & $61.9 \pm 13.5$ & $<0.001^{\star}$ \\
\hline Male sex & $32(82.1)$ & $142(49.3)$ & $<0.001^{\star}$ \\
\hline Body height $(\mathrm{cm})$ & $162.9 \pm 8.8$ & $162.3 \pm 9.8$ & 0.135 \\
\hline Body weight (kg) & $65.6 \pm 10.8$ & $60.9 \pm 13.3$ & $0.035^{\star}$ \\
\hline Waist circumference $(\mathrm{cm})$ & $85.5 \pm 10.8$ & $82.7 \pm 10.1$ & 0.122 \\
\hline Body mass index $\left(\mathrm{kg} / \mathrm{m}^{2}\right)$ & $24.8 \pm 4.1$ & $23.0 \pm 3.7$ & $0.006^{*}$ \\
\hline$<25$ & $23(59.0)$ & $213(74.0)$ & 0.102 \\
\hline $25,<30$ & $13(33.3)$ & $66(22.9)$ & \\
\hline$>30$ & $3(7.7)$ & $9(3.1)$ & \\
\hline Smoking & $17(43.6)$ & $99(34.4)$ & 0.286 \\
\hline Nonsmoking & $22(56.4)$ & $189(65.6)$ & \\
\hline Alcohol-dinking & $25(64.1)$ & $161(55.9)$ & 0.485 \\
\hline Nonalcohol-drinking & $14(35.9)$ & $127(44.1)$ & \\
\hline Systolic blood pressure ( $\mathrm{mmHg}$ ) & $129.0 \pm 19.2$ & $121.6 \pm 17.8$ & $0.026^{*}$ \\
\hline Diastolic blood pressure ( $\mathrm{mmHg}$ ) & $73.5 \pm 11.6$ & $76.1 \pm 11.2$ & 0.24 \\
\hline Visceral fat area $\left(\mathrm{cm}^{2}\right)$ & $130.7 \pm 58.2$ & $89.0 \pm 48.1$ & $<0.001^{\star}$ \\
\hline $\mathrm{HDL}-\mathrm{C}(\mathrm{mg} / \mathrm{dL})$ & $53.2 \pm 13.6$ & $60.5 \pm 15.7$ & $0.006^{\star}$ \\
\hline $\mathrm{LDL}-\mathrm{C}(\mathrm{mg} / \mathrm{dL})$ & $117.3 \pm 31.0$ & $115.4 \pm 26.7$ & 0.693 \\
\hline $\mathrm{TG}(\mathrm{mg} / \mathrm{dL})$ & $103.9 \pm 45.0$ & $101.7 \pm 59.3$ & 0.82 \\
\hline Adiponectin $(\mu \mathrm{g} / \mathrm{mL})$ & $3.9 \pm 3.2$ & $4.9 \pm 3.7$ & 0.107 \\
\hline Fasting plasma glucose $(\mathrm{mg} / \mathrm{dL})$ & $112.8 \pm 23.4$ & $106.6 \pm 20.8$ & 0.083 \\
\hline $\operatorname{HbAlc}(\%)$ & $5.9 \pm 0.6$ & $5.8 \pm 0.7$ & 0.206 \\
\hline Insulin $(\mu \mathrm{U} / \mathrm{mL})$ & $7.3 \pm 5.0$ & $5.8 \pm 4.6$ & 0.074 \\
\hline HOMA-IR & $2.1 \pm 1.5$ & $1.6 \pm 1.4$ & 0.055 \\
\hline Urine microalbumin (mg/gCr) & $36.2 \pm 80.3$ & $14.4 \pm 60.1$ & $0.046^{\star}$ \\
\hline High sensitivity CRP (mg/dL) & $0.2 \pm 0.6$ & $0.1 \pm 0.3$ & 0.283 \\
\hline $\mathrm{BNP}(\mathrm{pg} / \mathrm{mL})$ & $28.2 \pm 32.7$ & $23.9 \pm 29.9$ & 0.402 \\
\hline Spinal epidural lipomatosis (\%) & 10.3 & 8.7 & 0.464 \\
\hline Metabolic syndrome (\%) & 28.9 & 16.0 & $0.045^{\star}$ \\
\hline
\end{tabular}

Values are presented as mean \pm standard deviation or number (\%) unless otherwise indicated.

DISH, diffuse idiopathic skeletal hyperostosis; HDL-C, high-density lipoprotein cholesterol; LDL-C, low-density lipoprotein cholesterol; TG, triglycerides; HbA1c, glycated hemoglobin; HOMA-IR, homeostasis model assessment as an index of insulin resistance; CRP, C-reactive protein; BNP, B-type natriuretic peptide. ${ }^{*} \mathrm{p}<0.05$, significant differences. 
and metabolic syndrome. In this model, we adjusted for confounding factors, including age, sex, and lifestyle factors such as smoking and alcohol drinking. VFA, blood pressure, lipid metabolism, and carbohydrate parameters were not used as adjustment factors because these factors were covered by the diagnostic criteria for metabolic syndrome. Data are expressed as mean \pm standard deviation. A p-value less than 0.05 was considered statistically significant. We used IBM SPSS Statistics ver. 22.0 (IBM Co., Armonk, NY, USA) and Stata 15 (Stata Corp., College Station, TX, USA) for all statistical analyses.

\section{RESULTS}

\section{Demographic Parameters and Metabolic Syndrome}

Demographic data pertaining to DISH and non-DISH groups are presented in Table 1. Patients in the DISH group were significantly older (DISH group, mean 74.3 years; non-DISH group, mean 61.9-years; $\mathrm{p}<0.001$ ), and the proportion of males was higher (DISH group, 82.1\%; DISH group, 49.3\%; $\mathrm{p}<0.001$ ). Mean body height, waist circumference, smoking habit, and alcohol consumption were similar between the 2 groups; by contrast, body weight (DISH group, $65.6 \pm 10.8 \mathrm{~kg}$; non-DISH group, $60.9 \pm 13.3 \mathrm{~kg} ; \mathrm{p}=0.035$ ) and BMI (DISH group, 24.8; non-DISH group, 23.0; $\mathrm{p}=0.006$ ) were significantly higher in the DISH group than in the non-DISH group.

\section{Blood Pressure and VFA}

Blood pressure and VFA on the abdominal CT were diagnostic criteria for metabolic syndrome. SBP in the DISH group (DISH group, $129 \pm 19.2 \mathrm{mmHg}$; non-DISH group, 121.6 \pm 17.8 $\mathrm{mmHg} ; \mathrm{p}=0.026$ ) was significantly higher than that in the nonDISH group, but DBP was comparable (Table 1). The VFA on abdominal CT was significantly larger in the DISH group than in the non-DISH group (DISH group, $130.7 \pm 58.2 \mathrm{~cm}^{2}$; non-
DISH group, $\left.89.0 \pm 48.1 \mathrm{~cm}^{2} ; \mathrm{p}<0.001\right)$.

\section{Blood and Urine Analyses}

Blood and urine analyses were conducted to evaluate lipid and carbohydrate metabolism, indicating metabolic abnormalities. In lipid metabolism parameters, no significant difference in LDL-C, TG, or adiponectin was observed between the 2 groups; however, HDL-C was significantly lower in the DISH group $(53.2 \pm 13.6 \mathrm{mg} / \mathrm{dL})$ than in the non-DISH group $(60.5 \pm 15.7$ $\mathrm{mg} / \mathrm{dL}, \mathrm{p}=0.006$ ) (Table 1). Similarly, carbohydrate metabolism parameters had no significant difference between the 2 groups except for urine microalbumin, which was higher in the DISH group (DISH group, $36.2 \pm 80.3 \mathrm{mg} / \mathrm{dL}$; non-DISH group, $14.4 \pm$ $60.1 \mathrm{mg} / \mathrm{dL} ; \mathrm{p}=0.046)$.

\section{Metabolic Syndrome and SEL on Lumbar MRI}

From the results of the investigated parameters and lumbar MRI, diagnoses of metabolic syndrome and SEL were made.

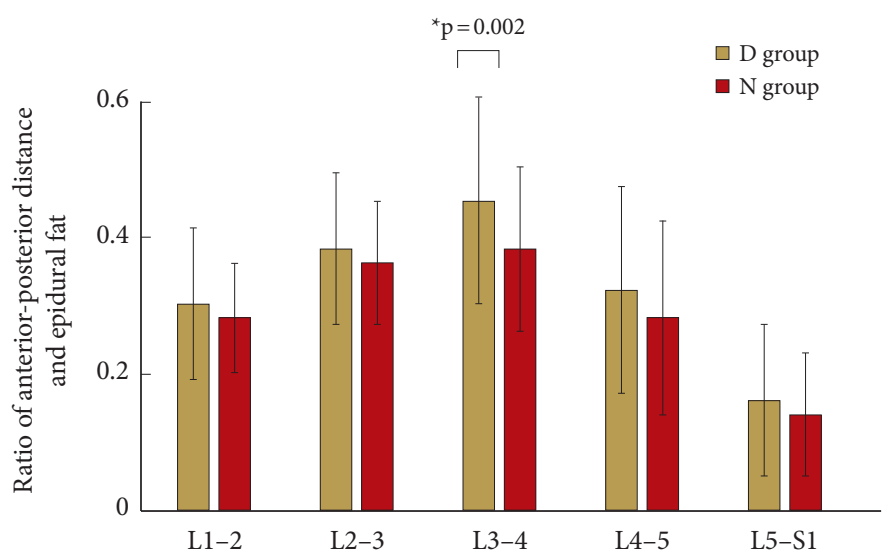

Fig. 1. Ratio of anterior-posterior distance and epidural fat from L1-2 to L5-S1. DISH, diffuse idiopathic skeletal hyperostosis; D group, DISH group; N group, non-DISH group. ${ }^{*} \mathrm{p}<0.05$, significant differences.

Table 2. Poisson regression model for the adjusted relative risk of DISH

\begin{tabular}{|c|c|c|c|c|c|c|c|c|}
\hline Variable & No. (\%) & $\begin{array}{l}\text { Prevalence } \\
\text { of DISH (\%) }\end{array}$ & $\begin{array}{l}\text { Relative risk } \\
\text { of DISH }\end{array}$ & $95 \%$ CI & p-value & $\begin{array}{l}\text { Adjusted relative } \\
\text { risk of DISH }\end{array}$ & $95 \%$ CI & $\mathrm{p}$-value \\
\hline \multicolumn{9}{|c|}{ Metabolic syndrome } \\
\hline Negative & $270(82.6)$ & 10.0 & Reference & & & Reference & & \\
\hline Positive & $57(17.4)$ & 19.3 & 1.9 & $1.0-3.7$ & 0.05 & 2.0 & $1.0-3.7$ & $0.04^{*}$ \\
\hline \multicolumn{9}{|c|}{ Spinal epidural lipomatosis } \\
\hline Negative & $298(91.1)$ & 10.4 & Reference & & & Reference & & \\
\hline Positive & $29(8.9)$ & 26.7 & 2.6 & $1.3-5.1$ & $<0.01^{\star}$ & 1.3 & $0.6-2.8$ & 0.54 \\
\hline
\end{tabular}

DISH, Diffuse idiopathic skeletal hyperostosis; CI, confidence interval. ${ }^{*} \mathrm{p}<0.05$, significant differences. 


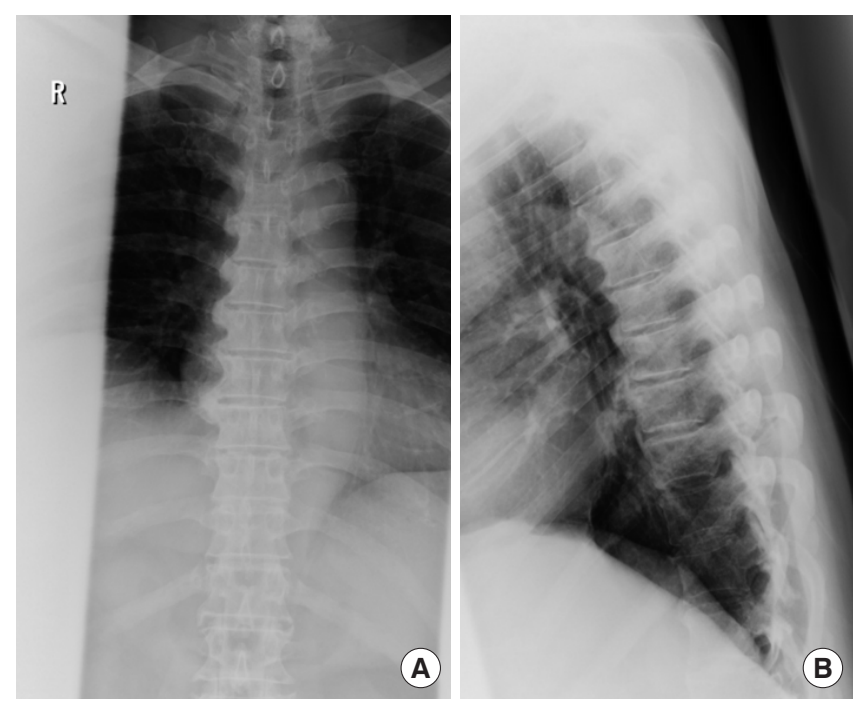

Fig. 2. Case presentation: a 73-year-old male. Serial radiographs of the thoracic spine indicated spinal ankylosis from T2 to T9. Anteroposterior (A) and lateral (B) radiographs.

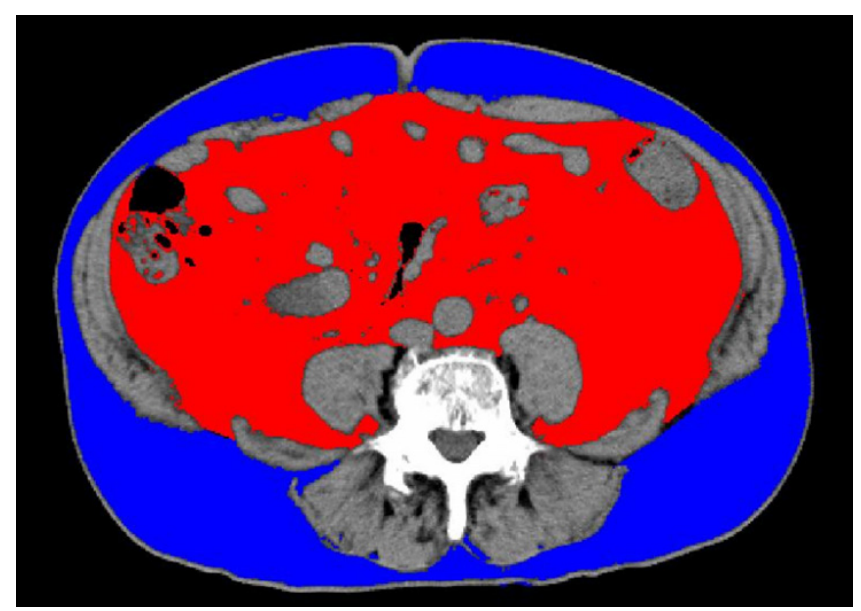

Fig. 3. Abdominal computed tomographic image of the visceral adipose area (red) and subcutaneous fat area (blue) at the level of the navel. The visceral adipose area was $258.1 \mathrm{~cm}^{2}$.

The prevalence of the metabolic syndrome was significantly higher in the DISH group (28.9\%) than in the non-DISH group (16.0\%, $\mathrm{p}=0.045)$. Based on lumbar MRI, 29 out of 327 subjects (8.9\%) were diagnosed with SEL. The prevalence of SEL was not significantly different between the 2 groups $(10.3 \%$ in the DISH group vs. $8.7 \%$ in the non-DISH group, $\mathrm{p}=0.464$ ). Fig. 1 illustrates the results of AP distance ratio of epidural fat at each level. At L3-4, the AP distance ratio was significantly larger in the DISH group $(0.45 \pm 0.11)$ than in the non-DISH group $(0.38 \pm 0.15 . p=0.002)$ (Fig. 1$)$. However, there was no significant difference at L1-2, L2-3, L3-4, or L5-S1.

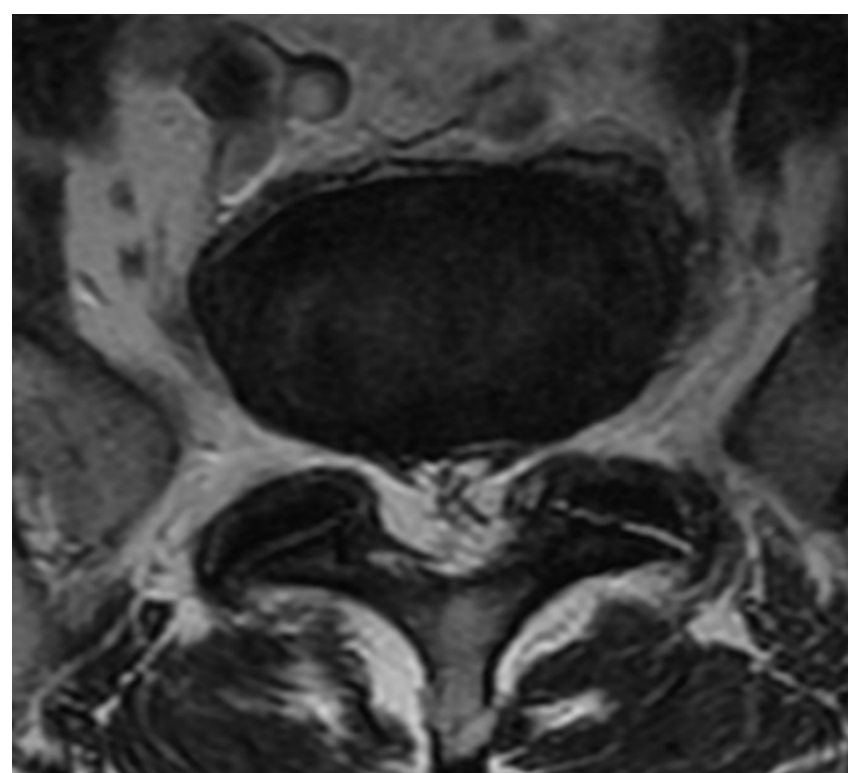

Fig. 4. The axial image of the lumbar magnetic resonance imaging showed anterior-posterior distance ratios for epidural fat of 0.61 at L5-S1.

\section{Subgroup Analysis}

We examined the association of metabolic syndrome and SEL with DISH (Table 2). Before adjustment for any variable, metabolic syndrome was weakly associated with DISH; however, SEL was significantly associated with DISH (relative risk [RR], 2.6; 95\% confidential interval [CI], 1.3-5.1; $\mathrm{p}<0.01$ ). After adjustment for age, sex, smoking habits, and alcohol drinking, metabolic syndrome was positively assciated with DISH (RR, 2.0; 95\% CI, 1.0-3.7; $\mathrm{p}=0.04$ ), but SEL was not associated with DISH. These results imply that the presence of DISH and metabolic syndrome exist together.

\section{Case Presentation}

A 73-year-old male had spinal ankylosis from T4-9 and was assigned to the DISH group (Fig. 2). His body height, body weight, and BMI were $162 \mathrm{~cm}, 78 \mathrm{~kg}$, and $29.4 \mathrm{~kg} / \mathrm{m}^{2}$, respectively. According to the abdominal CT image, the VFA was $258.1 \mathrm{~cm}^{2}$ and the patient had 2 positive conditions: (1) TG of $239 \mathrm{mg} / \mathrm{dL}$ and (2) SBP of $155 \mathrm{mmHg}$ and DBP of $99 \mathrm{mmHg}$ (Fig. 3). On the lumbar MRI, the AP distance ratios of epidural fat were lower than 0.60, except 0.61 at L5-S1 (Fig. 4). The patient was diagnosed with metabolic syndrome and SEL.

\section{DISCUSSION}

Here, we demonstrate that DISH was observed more frequent- 
ly in obese older males compared to the non-DISH subjects. These observations are consistent with those reported in previous studies. ${ }^{28,29}$ Toyoda et al. ${ }^{2}$ investigated findings from standing whole-spine radiographs of 345 consecutive patients who underwent spinal surgery and reported that the prevalence of DISH increased with age. In the present study, the prevalence of DISH was $11.9 \%$, relatively low. In 2018, Banno et al. ${ }^{29}$ evaluated 504 older volunteers and found that the prevalence of DISH was $8.3 \%$. Additionally, they found that DISH occurred more frequently in men (14.3\%) than in women (4.3\%), and that the mean age was higher in subjects with DISH than in those without DISH (78.4 years vs. 73.5 years, respectively). The prevalence of DISH is significantly affected by subject's population, such as patients who underwent spinal surgery ${ }^{2}$ or healthy volunteers. ${ }^{29}$ The all participants of the present study were medical checkup examinee without severe spinal complaint. Obesity is one of characteristics of DISH. Recently, Chaput et al. ${ }^{30}$ conducted CT-based survey of 214 trauma patients who were under 50 years of age and revealed that ossification of the anterior longitudinal ligament was associated with increased adiposity. Further, they suggested that obesity may play a more important role in spinal ossification than in age-related degeneration.

Our results indicate higher SBP and larger VFA in DISH subjects compared to non-DISH subjects, and one previous study ${ }^{31}$ has reported a higher frequency of hypertension in patients with DISH (61.1\%) compared to those without DISH (25.0\%). One of the highlights of the present study was estimation of the VFA in DISH subjects, which was significantly larger than that seen in the control group. Dan Lantsman et al. ${ }^{32}$ also investigated abdominal CT findings in 43 DISH patients and reported that VFAs were larger in DISH patients than in controls. They also suggested that the greater amount of fat tissue may represent a potential pathogenic pathway of enthesopathic excessive bone production. In 2001, Rogers and Waldron ${ }^{33}$ conducted a unique study to investigate the prevalence of DISH in skeletons from cemeteries in monasteries and churches. They found that skeletons from individuals with higher social status had a significantly greater prevalence of DISH compared to those from individuals with lower social status ( $\mathrm{p}<0.001)$; therefore, the authors opined that a rich diet, such as that with animal fat and alcohol, might be associated with the onset of DISH, even in medieval periods.

We revealed multiple abnormalities in laboratory data in subjects with DISH, such as in lipid (HDL-C) and carbohydrate (urine microalbumin) metabolism parameters. Further, the prevalence of DM in DISH patients tends to be greater than that in the normal population. ${ }^{18}$ In 2009, Mader and Lavi ${ }^{31}$ investigated individuals in the early phase of DISH, i.e., younger than 50 years of age, and reported that the prevalence of first-degree relatives with DM was greater in DISH patients. In 2018, they investigated serum adiponectin levels in patients with DISH and reported that higher serum adiponectin was associated with serum cholesterol and LDL levels and extension of bony bridges $(r=0.245, p=0.02)$. In the present study, lower HDL-C levels but higher urine microalbumin indicated abnormal carbohydrate and lipid metabolism in DISH subjects, suggesting that carbohydrate and lipid metabolism may play either a direct or an indirect role in the pathogenesis of DISH. Despite previous studies suggesting a relation between DISH and cardiovascular disease, ${ }^{20}$ we could not find any significant association.

The unique aspect of our study is that it analyzed the involvement of SEL in subjects with DISH. To the best of our knowledge, no previous study has investigated the association between DISH and SEL, and similarly to those in previous studies, the patient profile of subjects with SEL and DISH included obesity and metabolic dysfunction. ${ }^{26,34}$ Theyskens et al. ${ }^{34}$ reviewed the medical records of 28,902 patients and found that the factors associated with SEL were old age, high modified Charlson comorbidity index, male gender, BMI $>30 \mathrm{~kg} / \mathrm{m}^{2}$, and systemic corticosteroid use. In agreement with the Ishihara study, we found a larger amount of epidural fat on the lumbar MRI in DISH patients at all lumbar segments, especially a significant increase at L3-4. Our results indicate that systemic lipid metabolism abnormalities may exist in DISH subjects.

This study has several limitations. First, we diagnosed DISH based on serial radiographs rather than CT scans, even though CT scans can advantageously detect 3-dimensional vertebral ossification. We did not obtain spinal CT scans, but we hypothesize that CT scans of the whole spine would identify a larger prevalence of DISH than that reported here. Furthermore, we could not evaluate major ossification of the spine, ossification of posterior longitudinal ligament, because we could not obtain cervical spine radiographs. Second, as this was a cross-sectional study, a causal association between metabolic syndrome and DISH cannot be evaluated. Third, the subjects of the present study were not randomly selected healthy volunteers; rather, all subjects in the present study wanted information about their medical condition and thus agreed to participate in the medical checkup. This constitutes a potential selection bias. However, as there have been few studies investigating a possible association between metabolic syndrome and DISH, we believe that this study makes a significant contribution to the explanation of the 
etiology associated with ossification of the spinal ligaments.

In conclusion, our data suggested that advanced age, male sex, higher BMI, and metabolic syndrome are associated with DISH. Metabolic syndrome is a predisposing factor for DISH. This result can promote understanding the etiology of DISH.

\section{CONFLICT OF INTEREST}

MM has relevant financial activities outside the submitted work: NuVasive Japan (consultancy, institutional grant). The other authors declare that there are no relevant conflicts of interest.

\section{REFERENCES}

1. Resnick D, Shaul SR, Robins JM. Diffuse idiopathic skeletal hyperostosis (DISH): Forestier's disease with extraspinal manifestations. Radiology 1975;115:513-24.

2. Toyoda H, Terai H, Yamada K, et al. Prevalence of diffuse idiopathic skeletal hyperostosis in patients with spinal disorders. Asian Spine J 2017;11:63-70.

3. Schlapbach P, Beyeler C, Gerber NJ, et al. Diffuse idiopathic skeletal hyperostosis (DISH) of the spine: a cause of back pain? A controlled study. Br J Rheumatol 1989;28:299-303.

4. Aydin E, Akdogan V, Akkuzu B, et al. Six cases of Forestier syndrome, a rare cause of dysphagia. Acta Otolaryngol 2006; 126:775-8.

5. Okada E, Yoshii T, Yamada T, et al. Spinal fractures in patients with Diffuse idiopathic skeletal hyperostosis: a nationwide multi-institution survey. J Orthop Sci 2019;24:601-6.

6. Okada E, Shimizu K, Kato M, et al. Spinal fractures in patients with diffuse idiopathic skeletal hyperostosis: clinical characteristics by fracture level. J Orthop Sci 2019;24:393-9.

7. Okada E, Tsuji T, Shimizu K, et al. CT-based morphological analysis of spinal fractures in patients with diffuse idiopathic skeletal hyperostosis. J Orthop Sci 2017;22:3-9.

8. Okada E, Yagi M, Fujita N, et al. Lumbar spinal canal stenosis in patients with diffuse idiopathic skeletal hyperostosis: Surgical outcomes after posterior decompression surgery without spinal instrumentation. J Orthop Sci 2019;24:9991004.

9. Otsuki B, Fujibayashi S, Takemoto M, et al. Diffuse idiopathic skeletal hyperostosis (DISH) is a risk factor for further surgery in short-segment lumbar interbody fusion. Eur Spine J 2015;24:2514-9.

10. Yamada K, Satoh S, Abe Y, et al. Diffuse idiopathic skeletal hyperostosis extended to the lumbar segment is a risk factor of reoperation in patients treated surgically for lumbar stenosis. Spine (Phila Pa 1976) 2018;43:1446-53.

11. Westerveld LA, van Ufford HM, Verlaan JJ, et al. The prevalence of diffuse idiopathic skeletal hyperostosis in an outpatient population in The Netherlands. J Rheumatol 2008;35: 1635-8.

12. Kagotani R, Yoshida M, Muraki S, et al. Prevalence of diffuse idiopathic skeletal hyperostosis (DISH) of the whole spine and its association with lumbar spondylosis and knee osteoarthritis: the ROAD study. J Bone Miner Metab 2015;33: 221-9.

13. Weinfeld RM, Olson PN, Maki DD, et al. The prevalence of diffuse idiopathic skeletal hyperostosis (DISH) in two large American Midwest metropolitan hospital populations. Skeletal Radiol 1997;26:222-5.

14. Gerber LH, Helfgott RK, Gross EG, et al. Vertebral abnormalities associated with synthetic retinoid use. J Am Acad Dermatol 1984;10(5 Pt 1):817-23.

15. Kim SK, Choi BR, Kim CG, et al. The prevalence of diffuse idiopathic skeletal hyperostosis in Korea. J Rheumatol 2004; 31:2032-5.

16. Sukahara S, Miyazawa N, Akagawa H, et al. COL6A1, the candidate gene for ossification of the posterior longitudinal ligament, is associated with diffuse idiopathic skeletal hyperostosis in Japanese. Spine (Phila Pa 1976) 2005;30:2321-4.

17. Mader R, Novofestovski I, Adawi M, et al. Metabolic syndrome and cardiovascular risk in patients with diffuse idiopathic skeletal hyperostosis. Semin Arthritis Rheum 2009; 38:361-5.

18. Sencan D, Elden H, Nacitarhan V, et al. The prevalence of diffuse idiopathic skeletal hyperostosis in patients with diabetes mellitus. Rheumatol Int 2005;25:518-21.

19. Vezyroglou G, Mitropoulos A, Antoniadis C. A metabolic syndrome in diffuse idiopathic skeletal hyperostosis. A controlled study. J Rheumatol 1996;2:672-6.

20. Julkunen H, Aromaa A, Knekt P. Diffuse idiopathic skeletal hyperostosis (DISH) and spondylosis deformans as predictors of cardiovascular diseases and cancer. Scand J Rheumatol 1981;10:241-8.

21. Eckel RH, Grundy SM, Zimmet PZ. The metabolic syndrome. Lancet 2005;365:1415-28.

22. Alexander CM, Landsman PB, Teutsch SM, et al. NCEP-defined metabolic syndrome, diabetes, and prevalence of coronary heart disease among NHANES III participants age 50 years and older. Diabetes 2003;52:1210-4. 
23. Ford ES, Giles WH, Mokdad AH. Increasing prevalence of the metabolic syndrome among u.s. Adults. Diabetes Care 2004;27:2444-9.

24. Fujita N, Ishihara S, Michikawa T, et al. Potential association of metabolic and musculoskeletal disorders with lumbar intervertebral disc degeneration: cross-sectional study using medical checkup data. J Orthop Sci 2020;25:384-8.

25. Ishihara S, Fujita N, Azuma K, et al. Spinal epidural lipomatosis is a previously unrecognized manifestation of metabolic syndrome. Spine J 2019;19:493-500.

26. Fujita N, Hosogane N, Hikata T, et al. Potential involvement of obesity-associated chronic inflammation in the pathogenesis of idiopathic spinal epidural lipomatosis. Spine (Phila Pa 1976) 2016;41:E1402-7.

27. Definition and the diagnostic standard for metabolic syndrome--Committee to Evaluate Diagnostic Standards for Metabolic Syndrome. Nihon Naika Gakkai Zasshi 2005;94: 794-809.

28. Sirasanagandla SR, Al Dhuhli H, Al Abri A, et al. Prevalence of diffuse idiopathic skeletal hyperostosis among elderly subjects referred for radiological investigation in tertiary hospi- tal at Oman. Anat Cell Biol 2018;51:174-9.

29. Banno T, Togawa D, Hasegawa T, et al. The controlled study of diffuse idiopathic skeletal hyperostosis for the assessment of physical function in elderly populations. J Orthop Sci 2018; 23:929-34.

30. Chaput CD, Siddiqui M, Rahm MD. Obesity and calcification of the ligaments of the spine: a comprehensive CT analysis of the entire spine in a random trauma population. Spine J 2019;19:1346-53.

31. Mader R, Lavi I. Diabetes mellitus and hypertension as risk factors for early diffuse idiopathic skeletal hyperostosis (DISH). Ost eoarthritis Cartilage 2009;17:825-8.

32. Dan Lantsman C, Herman A, Verlaan JJ, et al. Abdominal fat distribution in diffuse idiopathic skeletal hyperostosis and ankylosing spondylitis patients compared to controls. Clin Radiol 2018;73:910.e15-910.e20.

33. Rogers J, Waldron T. DISH and the monastic way of life. Int J Osteoarchaeol 2001;11:357-65.

34. Theyskens NC, Paulino Pereira NR, Janssen SJ, et al. The prevalence of spinal epidural lipomatosis on magnetic resonance imaging. Spine J 2017;17:969-76. 